\title{
Full waves, empty waves and subquantum processes
}

\section{V.A. Skrebnev ${ }^{1}$ and M.V. Polski ${ }^{2}$}

1 Institute of Physics, Kazan Federal University, 18 Kremlyovskaya str., Kazan 420008, Russia

email: vskrebnev@gmail.com

2 East-West University, 816 S. Michigan Ave., Chicago, IL 60605, USA

\begin{abstract}
The appearance of a particle in a certain point in space, the full waves and the empty waves are considered as a consequence of subquantum processes. The experiment is described that measured the absorption of single photons by absorbers with various absorption coefficients, in one of the beams, after the photons interacted with the beam splitter. The measurements showed that the absorption corresponds to single photon traveling in either one or another beam. The results of our measurements and of single photon interference experiments, combined together, demonstrate the existence of the empty waves, that is, the excitations in the subquantum world, which do not contain a photon. We show that seemingly justified criticism of our interpretation of the experiment is not valid. New experiments are proposed to study single-photon interference involving an empty wave.
\end{abstract}

Key words: pilot wave, full wave, empty wave, subquantum processes, Copenhagen interpretation, single photon experiment, de Broglie-Bohm theory, 


\section{Introduction}

In 1986 Grangier, Roger and Aspect [1] have demonstrated the interference of the two output beams from the beam splitter in experiments using single photon states, even though the photon can only be detected in one of the two output beams for a given run of the experiment. Paper [2] considers the supposition that one of the beams contains a wave which is not accompanied by a particle, i.e. an empty wave. This supposition is consistent with the de Broglie - Bohm theory.

According to the de Broglie-Bohm theory it can be assumed that while a single photon travels in one particular beam, the so-called "pilot wave" that influences it travels in both. Thus, the empty wave can be considered part of the pilot wave. A number of works suggest experiments for the detection of empty waves (see, e.g. [3-5]); however, until now no experimental evidence of the existence of such wave has been received.

The Born rule connects the wave function to the probability density of finding the particle at a given point. However, this rule is not a basic law. In de Broglie-Bohm theory the wave is considered a physical reality, and the link between the probability density and the wave function has the status of a hypothesis. This means that the wave function may be not equal to zero even in the part of space where the particle is not observed. Hence the empty wave may also be described by the wave function, which may explain the interference of the wave containing the photon, i.e. the probability wave, or full wave, and the empty wave.

Clearly in order to discuss the empty wave it is important to understand the physical meaning of the wave function, i.e. to have an adequate interpretation of the wave function. We will not discuss all interpretations of wave function here. The fact that so many exist testifies to the absence of a satisfactory one. This is why many physicists lean towards the instrumentalist interpretation, best summed up in the succinct slogan "Shut up and calculate!" [6]. 
In our view, for a particle to be found in a point in space, it must actually be there at the moment when it is found. It is clear that for a particle to be manifested at a point in space, physical processes are needed to provide for this manifestation. In papers $[7,8]$ we offer an interpretation of the wave function in which wave function is some mathematical representation of real physical processes taking place at the subquantum level of the organization of matter which underlies the phenomena described by quantum mechanics.

The wave function is a function of coordinates that relate at any given time to the entire interval on which the function is defined. Most likely, this fact provoked the emergence of the Copenhagen interpretation of quantum mechanics, which rejects questions like "where was the particle before I registered its location" as meaningless. However, the inability of the wave function to specify the location of the particle at a particular time does not in any way imply the meaninglessness of the question about this location. We believe that at least before the Copenhagen interpretation particles existed in nature independently of observation. We are convinced that the particle is located in a specific place at a specific time, where it is detected during observation. From our point of view, the domain of the wave function corresponds to the domain of excitation in the subquantum world, in which subquantum processes can manifest a particle. In accordance with the Born rule, the wave function allows us to determine the probability of this manifestation at a certain point. We emphasize that the manifestation of a particle at some point in the domain does not mean that there is no subquantum excitation in the rest of the domain space. In advance, it is impossible to exclude the existence of a region of subquantum excitation associated with a particle, where subquantum processes do not manifest the particle itself. Given the absence of a particle, this area can be called empty. Accordingly, the subquantum excitation wave, in which the particle is present (the de Broglie pilot wave), can be called the probability wave or the full wave, and the excitation wave in which the particle is absent can be called the empty wave.

The pilot wave and the empty wave are created by real subquantum processes, that is, they are physical reality, not just mathematical objects. We think that the road into the 
structure of matter is a staircase with an infinite number of steps, and the subquantum level is one of these steps.

Experiments observe the manifestation of the particle in a certain point of the probability wave. It is common knowledge that behind every chance there is a rule. We do not know the rules of the subquantum world determining the formation of wave trains in which the particle manifests as an observable object. It is clear, however, that the photon can manifest anywhere in the wave train, and that this possibility is determined by some subquantum processes. A direct proof of the existence of the empty wave would mean that along with the wave train where the photon manifests, there also exists a wave train containing the potential possibility of the photon manifestation, but the actual photon does not manifest, and thus cannot be detected. Note that at any given time the photon manifests only in one of the points of the probability wave train. In all other points at that moment the wave may be considered empty.

According to [1], after the recombination of two beams the interference picture is observed. This implies that two coherent wave trains appear as a result of the interaction of the photon with the beam splitter. If the photon were to manifest in both wave trains, it would mean that its wave function is the superposition of two probability waves: the transmitted one and the reflected one. The empty wave hypothesis is consistent with the suggestion that the photon manifests in only one of the two output beams. In this case the photon wave function cannot be a superposition of two probability waves. Thus, experiments are needed which will clarify whether the photon wave function after the interaction with the beam splitter is the superposition of two probability waves or the superposition of a probability wave and an empty wave.

\section{Experiment and discussion}

Our experiment is illustrated in figure 1. 


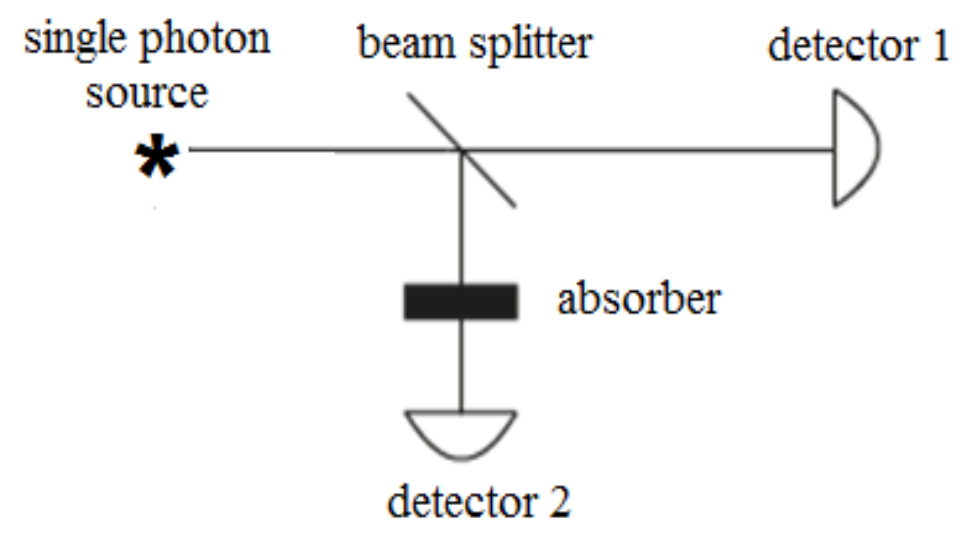

Fig.1

Let us consider that after the beam splitter the wave function of the single photon is the superposition of the wave functions corresponding to the transmitted and the reflected probability waves:

$$
\psi=c_{1} \psi_{t r}+c_{2} \psi_{r e}
$$

where the ratio of $\left|c_{1}\right|^{2}$ and $\left|c_{2}\right|^{2}$ is equal to the ratio of the numbers of photons registered by the detectors 1 and 2 in the absence of the absorber. Let $N_{0}$ is the number of the photons registered by both detectors in the absence of the absorber. Then numbers of the photons registered by the detector 1 and by the detector 2 will be equal to $N_{0}\left|c_{1}\right|^{2}$ and $N_{0}\left|c_{2}\right|^{2}$ accordingly.

If the number of the absorbed photons is $N_{a b}$, then the number of the unabsorbed photons is $\left(N_{0}-N_{a b}\right)$. Photon absorption has no effect on the wave function (1) of the unabsorbed photon after the beam splitter. If the wave function of the single photon were the superposition (1) and the absorber does not influence unabsorbed photons, then at the presence of the absorber the numbers of the photons registered by the detector 1 and by the detector 2 would be equal to $\left(N_{0}-N_{a b}\right)\left|c_{1}\right|^{2}$ and to $\left(N_{0}-N_{a b}\right)\left|c_{2}\right|^{2}$. The numbers of the photons registered by the detectors 1 and 2 would diminish on $N_{a b}\left|c_{1}\right|^{2}$ and $N_{a b}\left|c_{2}\right|^{2}$ accordingly.

The measurement results are provided in Table 1. 


\begin{tabular}{|l|l|l|l|l|l|}
\hline $\mathrm{k}$ & $\mathrm{T}$ & \multicolumn{1}{|c|}{$N_{1}$} & $N_{2}$ & $N_{1} / N_{2}$ & $\left(N_{1} / N_{2}\right)_{a v}$ \\
\hline 0 & 2 & 44730 & 33745 & 1.325 & \\
\hline 0 & 0.02 & 459 & 343 & 1.339 & 1.346 \\
\hline 0 & 0.002 & 45 & 33 & 1.373 & \\
\hline 0.1 & 2 & 44271 & 26068 & 1.698 & \\
\hline 0.1 & 0.02 & 433 & 255 & 1.696 & 1.701 \\
\hline 0.1 & 0.002 & 43 & 25 & 1.710 & \\
\hline & & & & & \\
\hline 0.2 & 2 & 44188 & 20502 & 2.155 & \\
\hline 0.2 & 0.02 & 446 & 210.6 & 2.118 & 2.096 \\
\hline 0.2 & 0.002 & 44 & 28 & 2.016 & \\
\hline
\end{tabular}

Table 1. Column names:

$\mathrm{k}$ - absorption coefficient of the absorber

$\mathrm{T}=20 T_{m}$

$T_{m} \quad$ - duration of one measurement cycle

$N_{1}$ - average number of photons registered by detector 1 in one measurement cycle $N_{2}$ - average number of photons registered by detector 2 in one measurement cycle $\left(N_{1} / N_{2}\right)_{a v}$ - average ratio of the number of photons registered by the detector 1 and the detector 2 at different $\mathrm{T}$ 
The table 1 shows that the ratio of the number of the photons registered by the detector 1 and the detector 2 without the absorber is 1.346 (with variation in the third decimal at various values of T). This means, that in the absence of the absorber the number of the photons registered by the detector 1 is

$$
\left(N_{1}\right)_{0}=0.574 N_{0}
$$

and the number of the photons registered by the detector 2 is

$$
\left(N_{2}\right)_{0}=0.426 N_{0}
$$

Let us suppose that after the beam splitter the wave function of the single photon is the superposition

$$
\begin{gathered}
\psi=c_{1} \psi_{t r}+c_{2} \psi_{r e} \\
\left|c_{1}\right|^{2}+\left|c_{2}\right|^{2}=1
\end{gathered}
$$

where $\left|c_{1}\right|^{2}=0.574,\left|c_{2}\right|^{2}=0.426$.

Let us admit that the absorber does not influence unabsorbed photon and the photon wave function remains the superposition (4). Then, when absorber is present, the number of the photons registered by the detector 1 would be equal to $0.574\left(N_{0}-N_{a b}\right)$ and the number of the photons registered by the detector 2 would be equal to $0.426\left(N_{0}-N_{a b}\right)$. The ratio of the numbers of the photons registered by the detectors 1 and 2 would remain equal to the ratio 0.574 and 0.426 , i.e. equal to 1.346 . Our measurements have shown, however, that the presence of the absorber in the path of the reflected beam does not change the number of the photons registered by the detector 1 . In the presence of the absorber, the number of unabsorbed photons registered by the detector 2 corresponds up to the third decimal to the expression

$$
N_{2}=\left(N_{2}\right)_{0} 10^{-k}
$$

Because the presence of the absorber does not change the number of the photons registered by the detector 1 , the number of absorbed photons is equal to

$$
N_{a b}=\left(N_{2}\right)_{0}-N_{2}=\left(N_{2}\right)_{0}\left(1-10^{-k}\right)=0.426 N_{0}\left(1-10^{-k}\right)
$$


In the presence of the absorbers the ratio of the numbers of photons registered by the detectors 1 and 2 also up to the third decimal corresponds to the expression

$$
\frac{N_{1}}{\left(N_{2}\right)_{0} 10^{-k}}=1.346 * 10^{k}
$$

so it does change.

Let us suppose that the photon wave function after the beam splitter is the superposition (4) and the absorber influences unabsorbed photon. Let us suppose also that as a result of the absorber influence the photon appears in the reflected beam (like an electron appears under influence of light in two slits experiments [9]). This photon will be registered by the detector 2 . Obviously, it may not increase the number of the photons registered by the detector 1 and to do it equal to $N_{0}\left|c_{1}\right|^{2}$ ).

Let's admit now that the tricky absorber transforms the wave function of an unabsorbed photon (4) into a superposition

$$
\psi=c_{t r} \psi_{t r}+c_{r e} \psi_{r e}
$$

where

$\left|c_{t r}\right|^{2}=\frac{N_{0}\left|c_{1}\right|^{2}}{N_{0}-N_{a b}}$ and $\left|c_{r e}\right|^{2}=\frac{N_{0}\left|c_{2}\right|^{2}-N_{a b}}{N_{0}-N_{a b}}$

In this case, the number of photons recorded by detectors 1 and 2 will be equal to $\left(N_{0}-N_{a b}\right)\left|c_{t r}\right|^{2}=N_{0}\left|c_{1}\right|^{2}$ and $\left(N_{0}-N_{a b}\right)\left|c_{r e}\right|^{2}=N_{0}\left|c_{2}\right|^{2}-N_{a b}$, as in our experiment. However, it is obvious. that the real absorber is not able to perform such transformation.

Thus, it is not possible to explain our results based on superposition (4). The results of our measurements, reflected in formulas (2), (5) and (7), correspond to the photon traveling after the beam splitter in either one or another beam.

Let us now discuss the seemingly justified criticism of our interpretation of the experiment. Let us consider the experiment proposed by critics that uses a second beam splitter instead of the absorber (Fig.2). 


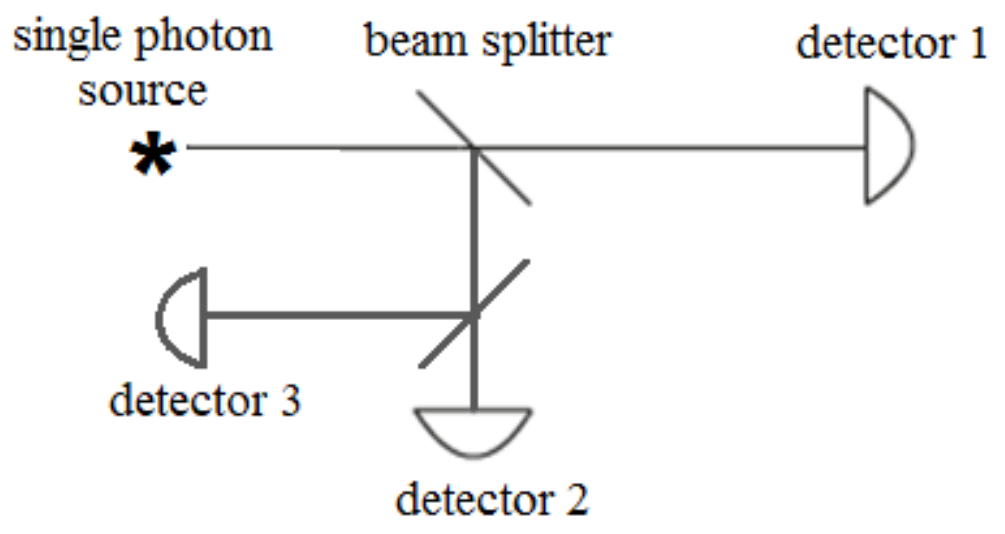

Fig.2

If we assume that after the second beam splitter we have a superposition of the wave $\psi_{\operatorname{tr}(2)}$ that passed this beam splitter and the wave $\psi_{r e(2)}$ reflected from it, then the wave function of the photon can be represented as

$$
\psi=c_{1} \psi_{t r}+c_{1(2)} \psi_{\operatorname{tr}(2)}+c_{2(2)} \psi_{r e(2)}
$$

Let the ratio $\left|c_{1(2)}\right|^{2}$ and $\left|c_{2(2)}\right|^{2}$ be equal to the ratio of the number of photons recorded by the detector 2 and the number of photons absorbed in our experiment. Then the number of photons recorded by the detectors 1 and 2 in the experiment with two beam splitters will be equal to the number of photons recorded by the detectors 1 and 2 in our experiment. The number of photons recorded by the detector 3 will be equal to the number of photons absorbed in our experiment. From this fact critics conclude that the experiment with two beam splitters is equivalent to our experiment. The results of measurements in the experiment with two beam splitters can be interpreted in the traditional way based on the superposition (1). Therefore, according to critics, the results of our measurements can also be interpreted without involving the idea of the empty wave.

If our experiment were equivalent to the experiment in which the absorber is replaced by a second beam splitter, then we could not claim that the results of our measurements indicate the existence of an empty wave. But these experiments could be considered equivalent only if the photon wave function in our experiment were a superposition of the 
functions $\psi_{t r}$ and $\psi_{\operatorname{tr}(2)}$ corresponding to the waves passed beam splitter and absorber, as well as some wave function $\varphi$ corresponding to the absorbed photon, that is if

$$
\psi=c_{1} \psi_{t r}+c_{1(2)} \psi_{t r(2)}+c_{2(2)} \varphi
$$

However according to the basic principles of quantum mechanics the wave function $\Psi$ can be considered the superposition

$$
\Psi=\sum_{n} c_{n} \psi_{n}
$$

of wave functions $\psi_{n}$ only if the functions $\psi_{n}$ are eigenfunctions of the same operator. As a result of the absorption of a photon, a state of the substance of the absorber occurs, which can be described by a certain wave function $\varphi$. However, there is no operator whose eigenfunctions are both the function $\psi_{\operatorname{tr}(2)}$ and the function $\varphi$. Accordingly, we do not have the right to approve that the absorber transforms the photon wave function into the superposition of the wave functions $\psi_{\operatorname{tr}(2)}$ and $\varphi$. This means that superposition (9) does not exist.

If in the experiment with two beam splitters the wave function is described by superposition (8), then before absorption by one of the detectors the photon belongs concurrently to all three beams. In our experiment, we can't claim that the photon belongs concurrently to the transmitted beam, the reflected beam and the absorber. Detectors record only unabsorbed photons whose number is equal to $N_{0}-N_{a b}$. Therefore, it is impossible to consider our experiment equivalent to the experiment with two beam splitters.

The results of measurements in the experiment with two beam splitters can be interpreted both on the basis of the superposition (1) and under the assumption the photon traveling after the beam splitters in either one or another beam. If there were a superposition (9), then the results of our experiment could also be explained both on the basis of the traditional approach and under the assumption the photon traveling only in one of the possible beams. However, due to the fact that superposition (9) does not exist, 
it is impossible to explain the results of our measurements on the basis of the traditional approach.

\section{Conclusion}

Our measurements show the photon traveling after the beam splitter in either one or another beam. At the same time, for the interference in the experiments [1] to be observed, two waves must be superposed. This means that while the photon is traveling in one beam, the empty wave is traveling in the other beam. In other words, the wave function of the photon after the interaction with the beam splitter is the superposition of the probability wave (full wave) and the empty wave.

The need to perform further experiments is obvious. For example, similar double-slit experiments are possible. It would be very interesting to study single-photon interference in the presence of an absorber. If the absorber does not absorb the empty wave, then the interference pattern will be created by photons passed the free beam and corresponding empty waves, as well as photons passed through the absorber and their empty waves. Otherwise, the interference pattern can only be created by photons passed the absorber. We can consider also the case when the absorber affects the empty wave, but does not absorb it, as well as the case of complete absorption of photons by the absorber.

Quantum mechanics is a fundamental theory which allows to describe a vast number of physical phenomena. However, as a truly fundamental theory, it cannot explain and describe itself. The survey "Models of wave-function collapse, underlying theories, and experimental tests" [10] points out: "Quantum mechanics is an extremely successful theory... One should of course stay cautioned against assuming that quantum theory will be successful through and through ... The fact that a theory is extremely successful in one part of the parameter space should not be taken as a guarantee that it will continue to be successful in a different part of the parameter space ... And there are historical examples of long-standing successful theories eventually turning into approximations to more 
general theories when their extrapolation into a new part of the parameter space failed to be confirmed by experiment."

Quantum mechanics cannot specify the location of a particle, since it does not describe the subquantum processes that ensure the manifestation of a particle at a certain point in space. Also, quantum mechanics says nothing about subquantum processes which provide for the existence of the empty wave. Experimental proof of the existence of the empty wave may be useful in developing a more general theory, to which quantum mechanics will be an approximation.

\section{References}

[1] P. Grangier, G. Roger and A. Aspect, Europhys. Lett. 1 (1986) 173.

[2] L. Hardy, "On the existence of empty waves in quantum theory", Physics Letters A, 167 , issue 1 , page 11 (1992).

[3] J. R. Croca, “An experiment for detection of empty waves”, Physics Letters A, 124, no. 1,2 , page 22 , (1987)

[4] Peter E. Gordon, “Can empty waves be detected?”, Physics Letters A, 138, no. 8, page $359,(1989)$

[5] Sofia Wechsler, About empty waves, their effect, and the quantum theory, $\underline{\operatorname{arXiv}: 1008.4849 \mathrm{v} 1}$ [quant-ph]

[6] Mermin, D.: Could Feynman Have Said This? Physics Today 57 (5) 10-11 (2004)

[7] V.A. Skrebnev, M.V. Polski, Some issues of quantum theory and subquantum processes, arXiv:1701.03019v4

[8] Polski, M.V., Skrebnev, V.A., An alternative derivation of canonical distribution as a result of irreversible processes in macrosystems, Eur. J. Phys. 38, 025101 (2017)

[9] The Feynman Lectures on Physics, Volume III, Chapter 1

[10] A. Bassi, et all, Models of wave-function collapse, underlying theories, and experimental tests, Reviews of Modern Physics, 85, 471 (2013). 